\title{
Numerical Calculation for Effect of Fluid-structure Interaction on Flow Field Pressure in High-Flow Control Valve
}

\author{
Fang CAO \\ School of Mechanical and Automotive Engineering, Qilu University of Technology, Jinan 250353, \\ China \\ caofang03@163.com
}

Keywords: Fluid-structure Interaction (FSI), Flow field pressure, Pressure drop, High-flow control valve.

\begin{abstract}
According to the actual structure and working condition of a high-flow gas pressure control valve in cycle power generation project, 3-D model based on Fluid-structure Interaction (FSI) is set up. A combined calculation for turbulent flow and structure response of high-flow control valve was established, using two-way coupling method to study the effect of FSI of valve on flow field pressure. The results of flow field pressure and pressure drop are analysed, comparing with the calculated results without FSI. The analysis results indicate that, FSI has a significant effect on the flow field pressure and pressure drop of position on valve core. It cannot be ignored when studying a high-flow control valve. The 3-D simulation model and flow field pressure analysis based on FSI can provide an effective method for high-flow control valve research and design optimization.
\end{abstract}

\section{Introduction}

Lots of coke-oven and blast-furnace gas are produced in steelworks manufacturing. At present, in order to reduce the total energy loss and environmental pollution, many steelworks start generating electricity using waste gas to develop recycling economy. In the process of transporting gas, the influence of changes in gas turbine load, the mixed gas calorific value, the environment temperature, atmospheric pressure, and gas source supply, is bound to cause the gas pressure and velocity of unstable [1-2], which cause air flow pulsation.

However, the fuel gas electric generating set is used in recycling generating electricity project, which need input stable gas pressure [3-4]. The common problem of recycling generating electricity project is to achieve pressure stability of high-flow gas. The stability of the control valve is mostly depends on the gas flow inside the valve. It is closely related to the flow state [5]. Therefore, the study of gas flow and its pressure drop is very important to research on the valve stability.

The Fluid-structure Interaction (FSI) of gas with valve could influence the spatial distribution regularity of flow field inside the valve, inducing the coupling vibration, noise, also producing the supernormal pressure, stress, etc., which make the control valve produce various instability phenomenon, directly affecting the safety of work. So FSI of control valve for fluid pressure is an important premise of valve design. George Papadakis [6] proposed a new method to solve the problem of fluid-structure interaction, which can be used to predict pipeline pressure fluctuations; James A. Davis [7] et al. studied the effect of valve core shape on valve inherent flow characteristics through simulation and experiment methods. Pei Ji [8] established a combined calculation for turbulent flow and structure response of impeller using two-way coupling method to study the effect of FSI of impeller on flow field in centrifugal pump. ZENG Lifei et al. [9] performed numerical simulation for the effect of the control valve vibration on the flow field under the help of dynamic-mesh and UDF function of Fluent.

According to actual structure and working condition of high-flow control valve in cycle power generation project, the numerical calculation model of control valve based on FSI is established. The flow field pressure analysis is studied. 


\section{Model of Control Valve}

According to the way of installation and using, the 3-D high-flow control valve model is set up. Figure 1 depicts structure of control valve, which the nominal diameter is 10 inch. Figure 2 is the half of the valve at 50\% valve opening, which is cut away from plane of symmetry in order to understand the internal structure of valve. The valve core type is balanced plug. On the basis of FSI analysis, the runner of valve plug- seat- body is regarded as the simulation computed field, and the intact runner is depicted in figure 3. Figure 4 shows the assembly drawing of valve core and fluid domain. This paper would analyze the control valve flow field pressure and pressure drop, considering the influence of interaction of fluid and valve. According to the real work condition, the generating electricity project uses $48 \mathrm{MW}$ gas turbine set, which need input gas pressure is $2.35 \mathrm{MPa}$. The initial condition is determined by calculation condition. The turbulence model is described by $\mathrm{K}-\varepsilon$ two equations.

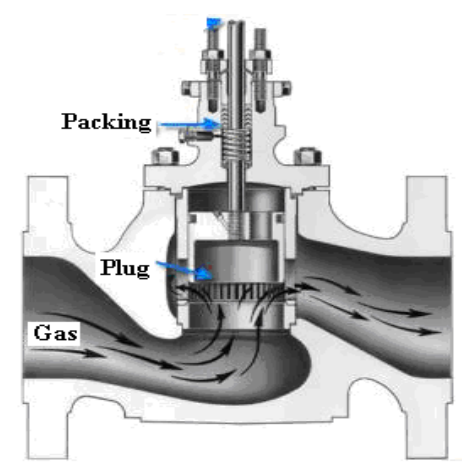

Fig. 1. Structure of control valve

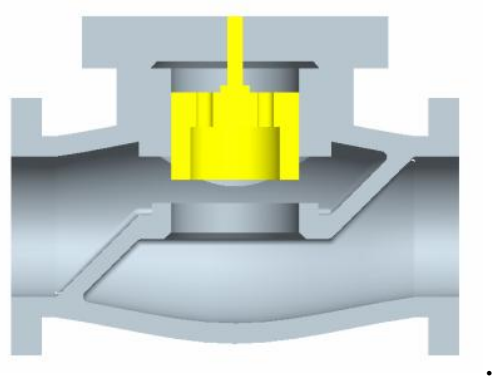

Fig. 2. The half of the valve

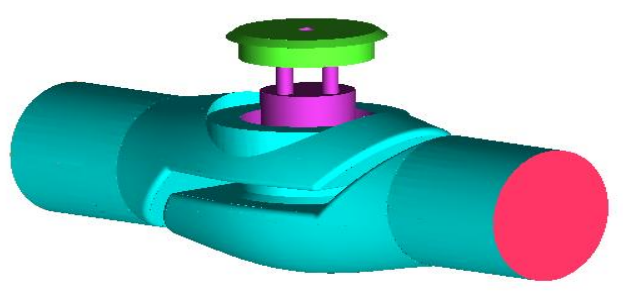

Fig. 3. The inner runner of valve

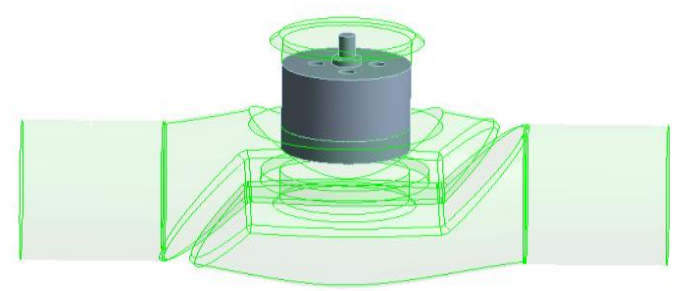

Fig. 4. The assembly drawing 


\section{Effect of FSI on Flow Field Pressure}

The pressure of flow field inside the high-flow control valve would change due to FSI. In order to be able to get the specific case of the effect of FSI on pressure and pressure drop, the valve port from inlet to outlet is divided into different section equally at $50 \%$ valve opening, as shown in the figure 5 . The position 1 is the entrance section and 11 is exit. The flow field pressure and pressure drop in these cross-sections are monitored considering FSI and not considering FSI respectively.

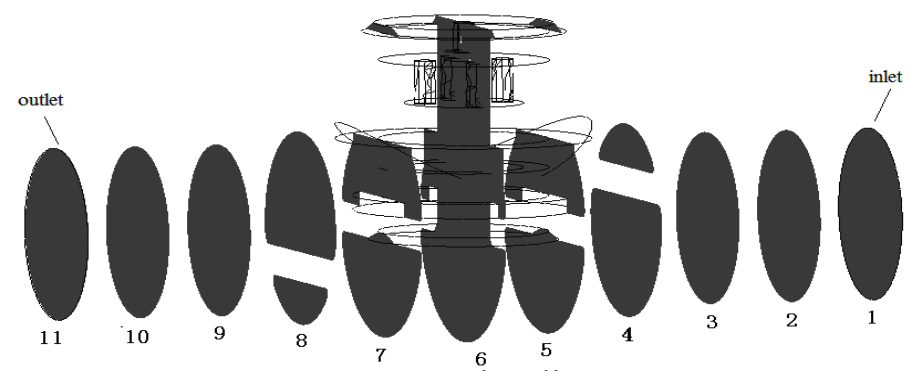

Fig. 5. The cross-section position

\section{Pressure analysis}

Through numerical simulation, the flow field pressure values of these cross-section positions are got. They are shown in table $1 . P 0$ representatives the flow field pressure without considering FSI, $p 1$ representatives the flow field pressure considering FSI. The percentage $(p 1-p 0) / p 0$ reflect the influence magnitude of FSI on the control valve flow field pressure. In order to compare the flow field pressure and of control valve at different positions more intuitively, they are depicted in figure 6.

Table 1. The flow field pressure

\begin{tabular}{|c|c|c|c|}
\hline Position & $\mathrm{p} 0(\mathrm{MPa})$ & $\mathrm{p} 1(\mathrm{MPa})$ & $(\mathrm{p} 0-\mathrm{p} 1) / \mathrm{p} 0(\%)$ \\
\hline 1 & 2.81214 & 2.73019 & 2.914 \\
\hline 2 & 2.81199 & 2.73002 & 2.915 \\
\hline 3 & 2.81041 & 2.72861 & 2.911 \\
\hline 4 & 2.74621 & 2.67092 & 2.742 \\
\hline 5 & 2.65013 & 2.6199 & 1.141 \\
\hline 6 & 2.59771 & 2.6482 & -1.944 \\
\hline 7 & 2.59831 & 2.56176 & 1.407 \\
\hline 8 & 2.4134 & 2.41092 & 0.103 \\
\hline 9 & 2.32428 & 2.33596 & -0.503 \\
\hline 10 & 2.34872 & 2.35311 & -0.187 \\
\hline 11 & 2.348 & 2.34908 & -0.046 \\
\hline
\end{tabular}

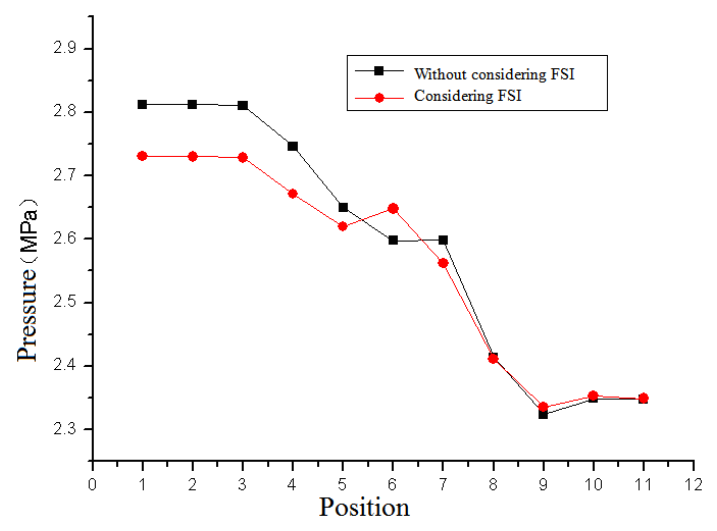

Fig. 6. Pressure of different positions 
It has an impact of FSI on pressure of different positions, especially in the cross section of position before 8 , as shown in figure 6 . Without considering FSI, the flow field pressure decreases from inlet to outlet. However, the pressure through valve core cross section, that is position 6 increases when considering FSI. It is even greater than the pressure value without FSI. The pressure valves at other cross section positions considering FSI are smaller or close to without considering FSI. It explains that FSI has a significant effect on valve core.

\section{Pressure drop analysis}

The pressure drop between adjacent slices from inlet to outlet is shown in table 2. $\triangle \mathrm{p} 0$ representatives the flow field pressure drop without considering FSI, $\triangle \mathrm{p} 1$ representatives the flow field pressure considering FSI. The percentage $(\triangle \mathrm{p} 1-\triangle \mathrm{p} 0) / \triangle \mathrm{p} 0$ reflect the influence magnitude of FSI on the control valve flow field pressure drop. In order to compare the pressure drop of control valve at different positions more intuitively, they are depicted in figure 7.

Whether considering FSI, the largest part of the pressure drop in the valve port is between 7 to 8 sections, from figure 7 . This is due to the cross-section 7 is located at the throttle mouth. As a result of the throttle action, a flow is created on the downstream side of the nearby place, which makes the speed of the throttling mouth section increase, but the pressure is reduced. So the pressure drop is the largest. After cross-section 8, along with the rising of the control valve flow area, the pressure is gradually restored, which is the reason of pressure drop decrease gradually. Of course, it already could not return to the original pressure value, which is also the main reason of the valve pressure difference. From the table 2, the greatest influence of FSI on the pressure drop is located in the section between 6 and 7, followed by the section between 5 and 6 , which are the sections near the valve core. Therefore, the effect of FSI on flow field pressure and the pressure drop of the valve core can't be ignored when analysis of control valve.

Table 2. The pressure drop

\begin{tabular}{|c|c|c|c|}
\hline Position & $\Delta \mathrm{p} 0(\mathrm{MPa})$ & $\Delta \mathrm{p} 1(\mathrm{MPa})$ & $(\Delta \mathrm{p} 0-\Delta \mathrm{p} 1) / \Delta \mathrm{p} 0(\%)$ \\
\hline $1-2$ & 0.00015 & 0.00017 & -13.333 \\
\hline $2-3$ & 0.00158 & 0.00141 & 10.759 \\
\hline $3-4$ & 0.0642 & 0.05769 & 10.140 \\
\hline $4-5$ & 0.09608 & 0.05102 & 46.898 \\
\hline $5-6$ & 0.05242 & -0.0283 & 153.987 \\
\hline $6-7$ & -0.0006 & 0.08644 & 14506.667 \\
\hline $7-8$ & 0.18491 & 0.15084 & 18.425 \\
\hline $8-9$ & 0.08912 & 0.07496 & 15.889 \\
\hline $9-10$ & -0.02444 & -0.01715 & 29.828 \\
\hline $10-11$ & 0.00072 & 0.00403 & -459.722 \\
\hline
\end{tabular}

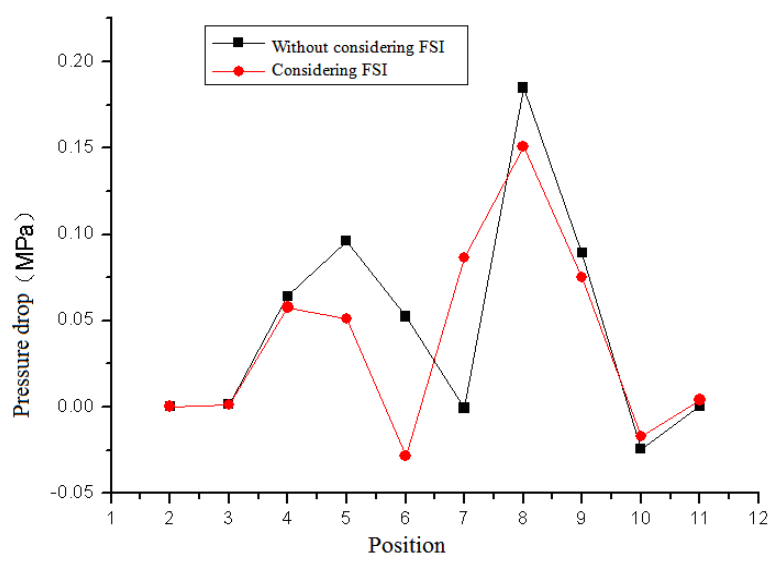

Fig. 7. Pressure drop of different positions 


\section{Conclusions}

In this work, 3-D model of control valve based on FSI is set up, according to the actual structure and working condition of the high-flow control valve in cycle power generation project. Flow field pressure analysis is carried out on the control valve coupling system. The valve port from inlet to outlet is divided into different section equally, and the pressure and pressure drop under the condition of coupled and uncoupled on these positions are compared, which proves the FSI effect on the flow field pressure. Considering FSI can greatly improve the accuracy of pressure analysis of control valve, especially on position of valve core. It has a significance of practical production to improve the designing research level of high-flow control valve.

\section{Acknowledgments}

This project is supported by Shandong Province Outstanding Research Award Fund for Young Scientists (BS2014ZZ016), and Shandong Province Key Project of Research and Development (2015GGH303001).

\section{References}

1. B Seyedan, P L Dhar, R R Gaur. et al, Computer Simulation of a Combined Cycle Power Plant, Heat Recovery System \& CHP, 15, 7, p.619-630(1995)

2. H Takano, Y Kitauchi and H Hiura. Design for the 145-MW Blast Furnace Gas Firing Gas Turbine combined Cycle Plant. ASME Journal of Engineering for Gas Turbines and Power, 111, 2, p.218-224, (1989)

3. J Y Shin, Y J Jeon, D J Maeng, et al. Analysis of the Dynamic Characteristics of a Combinedcycle Power Plant. Energy, 27, 12, p.1085-1098,(2002)

4. K Kunitomi, A Kurita, Y Tada, et al. Modeling Combined-cycle Power Plant for Simulation of Frequency Excursions. IEEE Transactions on Power Systems, 18, 2, p. 724-729(2003)

5. H.Y. PENG, D.B. YANG. Application and Effect of Combined Cycle Fuel Gas-Steam Turbine in Liangang. Metal Materials and Metallurgy Engineering, 37, 1, p. 46-49(2009)

6. George Papadakis. A novel pressure-velocity formulation and solution method for fluidstructure interaction problems. Journal of Computational Physics, 227, p. 3383-3404(2008)

7. James A. Davis. Predicting Globe Control Valve Performance-Part I; CFD Modeling. Transactions of the ASME, 124(2002)

8. Pei Ji; Yuan Shouqi; Yuan Jianping. Numerical Calculation for Effect of Fluid-structure Interaction on Flow Field in Centrifugal Pump. Journal of agricultural machinery, 40,12, p. 107112(2009)

9. ZENG Lifei, LIU Guanwei. MAO Jingru et al. Numerical Simulation for the Effect of the Control Valve Vibration on the Flow Field. Proceedings of the CSEE, 35,8, p. 1977-1982(2015) 